\title{
CD25 deficiency: A new conformational mutation prevents the receptor expression on cell surface
}

\author{
Marina Vignoli $^{\mathrm{a}, \mathrm{b}, 1}$, Sara Ciullini Mannurita ${ }^{\mathrm{a}, \mathrm{b}, 1}$, Antonella Fioravanti ${ }^{\mathrm{c}}$, Manuela Tumino ${ }^{\mathrm{d}}$, \\ Alessia Grassi $^{\mathrm{e}}$, Graziella Guariso ${ }^{\mathrm{d}}$, Claudio Favre ${ }^{\mathrm{b}}$, Mario M. D'Elios ${ }^{\mathrm{e}}$, Eleonora Gambineri ${ }^{\mathrm{a}, \mathrm{b}, *}$ \\ a Department of "NEUROFARBA", Section of Child's Health, University of Florence, Italy \\ b Department of Haematology-Oncology "Anna Meyer" Children's Hospital, Florence, Italy \\ ${ }^{\mathrm{c}}$ Department of Structural and Molecular Microbiology, Structural Biology Research Center, Structural Biology Brussels, VIB, Vrije Universiteit Brussel, Pleinlaan 2, 1050 \\ Brussels, Belgium \\ ${ }^{\mathrm{d}}$ Unit of Paediatric Gastroenterology, Department of Women's and Children's Health, University-Hospital of Padova, Padova, Italy \\ ${ }^{\mathrm{e}}$ Department of Experimental and Clinical Medicine, University of Florence, Italy
}

\section{A R T I C L E I N F O}

\section{Keywords:}

Primary Immune Deficiency (PID)

Immune Dysregulation

Polyendocrinopathy

Enteropathy

$\mathrm{X}$-linked (IPEX)

IPEX-like

Regulatory T cells (Treg)

Immunephenotyping

\begin{abstract}
A B S T R A C T
CD25 deficiency is a very rare autosomal recessive disorder that shows a clinical phenotype highly overlapping IPEX syndrome with an increased susceptibility to viral, bacterial, and fungal infections. It is due to mutations in the IL2R $\alpha$ gene that codes for the $\alpha$ subunit of the IL2 receptor complex.

Here we report the characterization of a novel $I L 2 R \alpha$ gene mutation leading to a severe protein conformational alteration that abrogates its cell surface expression in a child presenting with early-onset IPEX-like disorder. Cytofluorimetric analysis revealed the total absence of CD25 cell surface expression and addressed IL2R $\alpha$ molecular investigation.

The early clinical and molecular diagnosis of CD25 deficiency in this patient promptly led to hematopoietic stem cell transplantation (HSCT), allowing complete resolution of the symptoms and definitive cure of the disease.
\end{abstract}

\section{Introduction}

CD25 deficiency (OMIM \#606367) is a rare genetic disorder that shows an autosomal recessive inheritance. It was first described by Sharfe et al. in 1997 as an immunodeficiency characterized by increased susceptibility to viral, bacterial and fungal infections and decreased numbers of peripheral $\mathrm{T}$ cells with abnormal proliferation [1]. In 2007, Caudy et al. associated the deficiency of CD25 with IPEX-like phenotype since the reported patient showed clinical features resembling Immunedysregulation Polyendocrinopathy Enteropathy X-linked (IPEX) Syndrome caused by FOXP3 mutations, with a greater susceptibility to infections [2].

To date, only eight cases of six unrelated families have been reported (Supplementary information is available on EJHG's website, Table 2) [1-6]. All patients showed an early onset of symptoms and four of them presented with severe autoimmune enteropathy with histological findings of villous atrophy and several infections (usually viral and/or opportunistic) as common features. Four patients, including three patients of the same family, presented with autoimmunity, such as autoimmune cytopenias and diabetes as main clinical features. Five out of eight patients also developed severe eczema, while the presence of other autoimmune phenomena was more heterogeneous.

CD25 deficiency is due to mutations in the IL2R $\alpha$ gene (OMIM 147730; chr10p15.1) coding for the $\alpha$ subunit (CD25) of the IL2 receptor complex that, together with $\beta$ (CD122) and $\gamma$ (CD132) subunits, composes the high-affinity receptor for IL2. It has been suggested that $\mathrm{IL}-2 \mathrm{R} \alpha$ captures and concentrates free IL-2 at the cell surface for presentation to IL-2R $\beta$ and $\gamma c$ or that IL2/IL-2R $\alpha$ interaction induces conformational changes in IL-2 that stabilizes the formation of the

Abbreviations: IPEX, Immune dysregulation Polyendocrinopathy Enteropathy X-linked; PID, Primary Immune Deficiency; FOXP3, Forkhead box P3; HSCT, Hematopoietic Stem Cell Transplantation; Treg, Regulatory T cells; HM, Homology Modeling

*Corresponding author at: Department of "NEUROFARBA": Section of Child's Health, University of Florence, Department of Hematology-Oncology: BMT Unit, "Anna Meyer" Children's Hospital, Viale Gaetano Pieraccini,24, 50139 FIRENZE, Italy.

E-mail addresses: eleonora.gambineri@unifi.it, e.gambineri@meyer.it (E. Gambineri).

${ }^{1}$ These authors contributed equally to the work. 
ternary complex IL-2R $\alpha \beta \gamma$ [7]. CD25 is highly expressed on the surface of regulatory $\mathrm{T}$ cells (Tregs) that actively suppress pathological and physiological immune responses, thereby contributing to the maintenance of immunological self-tolerance and immune homeostasis [8]. The presence of CD25 enables Tregs to be the first responders to IL2 during an immune response [9] and allows the transcription of FOXP3 through the IL2/IL2R $\alpha$-STAT5b transduction pathway [10].

Here we report the characterization of a novel IL2R $\alpha$ gene mutation leading to a conformational alteration of protein subunits and consequential absence of cell surface expression in a patient presenting with early onset autoimmunity.

\section{Subjects and methods}

\subsection{Case report}

The patient was a two-month-old boy born to unrelated parents with uneventful pregnancy and delivery. At one month of age, he developed intractable watery diarrhoea that required parenteral nutrition (PN). Small bowel biopsies showed villous atrophy upon histopathological examination. Pseudomonas and Human herpesvirus 6 (HHV6) in stool were detected. He also developed severe dermatitis. Laboratory evaluation showed normal complete blood count, normal immunoglobulin serum levels (IgG $789 \mathrm{mg} / \mathrm{dl}$; IgA $150 \mathrm{mg} / \mathrm{dl} ; \mathrm{IgM} 260 \mathrm{mg} / \mathrm{dl}$ ) and mildly elevated IgE (169 kU/l). Immunophenotyping revealed a decrease of total lymphocytes in particular of naïve T cells, both CD4 (patient 44.5\%, healthy controls, HC 66-100\%) and CD8 (patient $55.7 \%$, HC 78.5-97.1\%), and a decrease of recent thymic emigrants (patient $23.6 \%$, HC 50-100\%). Total B cells were lower compared to HC (patient 5\%, HC 20.5-40.9\%) (Supplementary information is available on EJHG's website, Table 1).

\subsection{Flow cytometry}

Peripheral Blood Mononuclear Cells (PBMCs) of patient and HC were obtained by Ficoll-Paque PREMIUM (GE Healthcare, UK). Surface antibodies staining was performed for $15 \mathrm{~min}$ in the absence of light using a $0.5 \%$ bovine serum albumin PBS mixture. Cells were washed and treated with FOXP3 perm/fix buffer set (BioLegend, CA, USA) to be further stained for FOXP3. The following antibodies were used: CD4PerCp-Cy5.5 (SK3), CD25-PE (M-A251), CD127-AlexaFluor647 (HIL7R-M21), FOXP3-AlexaFluor488 (259D, BioLegend).

To evaluate CD25 intracytoplasmic expression, lymphocytes were stimulated with Dynabeads ${ }^{\circledast}$ CD3/CD28 $\mathrm{T}$ Cell Expander beads (Invitrogen, Thermo Fisher Scientific) and recombinant human IL-2 (rhIL-2, $100 \mathrm{U} / \mathrm{ml}$ ) (PeproTech ${ }^{\circledR}$, USA) and stained with antibodies to CD25-PE (2AE), CD4-APC (SK3), CD3-PE-Cy7 (SK7) and LIVE/DEAD ${ }^{\circledR}$ Fixable viability dye (Invitrogen, Thermo Fisher Scientific, Italy). Cells were fixed and permeabilized with FOXP3 perm/fix buffer and stained for intracytoplasmic CD25-FITC (M-A251).

All antibodies were purchased from BD Biosciences unless otherwise noted. Cells were acquired with FASCanto II and the analysis was performed with FACSDiva Software (BD Biosciences, USA).

\subsection{Proliferation assay and cytokine production}

Proliferation and cytokine production were investigated both in deficient patient and in HC, as described [11]. Polyclonal stimulation of PBMCs from HC and the patient was performed by culturing at $5 \times 10^{4}$ cells per well in duplicate in round-bottom 96-well tissue culture plates with medium, in presence of CD3/CD28 T Cell Expander alone or plus rhIL-2 in different concentrations $(10 \mathrm{U} / \mathrm{ml}$, or $100 \mathrm{U} / \mathrm{ml}$, or $1000 \mathrm{U} /$ $\mathrm{ml}$ ), or rhIL-15 $(10 \mathrm{ng} / \mathrm{ml})$ (R\&D system, UK). Furthermore $5 \times 10^{4}$ cells per well in duplicate were stimulated with the same stimulation media or with rhIL-2 $(100 \mathrm{U} / \mathrm{ml})$ plus rhIL-15 $(10 \mathrm{ng} / \mathrm{ml})$ medium without CD3/CD28 T Cell Expander. As negative control, cells were plated at $5 \times 10^{4}$ cells per well in duplicate with medium without stimuli. The cells were cultured for 3 days before pulsing with [3H]thymidine for $18 \mathrm{~h}$. Plates were harvested and proliferation was recorded as counts per minute (CPM) using a beta counter.

To assess cytokine production, $5 \times 10^{5}$ cells of the patient or the HC were stimulated with $0.5 \mathrm{ml}$ medium in presence or absence of PMA $10 \mathrm{ng} / \mathrm{ml}$ and ionomycin $1 \mu \mathrm{g} / \mathrm{ml}$ (Sigma-Aldrich) for $36 \mathrm{~h}$. At the end of the culture period, cell-free supernatants were collected and assayed for their cytokine content (IFN- $\gamma$, IL-4, IL-17) by ELISA (Invitrogen, Thermo Fisher Scientific, Italy).

\subsection{Molecular analysis}

Genomic DNA was isolated from peripheral blood leukocytes using the QIAamp DNA Blood Mini Kit according to the manufacturer's instructions (Qiagen, Hilden, Germany).

The entire coding sequence, including exon-intron junctions, was amplified using exon-specific primers (sequences available upon request) and standard PCR conditions. PCR products were sequenced using the BigDye Terminator Cycle Sequencing Kit (Applied Biosystems, Foster City, CA, USA) on an automated ABI PRISM 3130 Genetic Analyzer (Applied Biosystems) and compared to reported reference cDNA sequence (GenBank accession no. NM_000417.2). Written informed consent to genetic investigations, approved by the local ethics committee, was obtained from the patient's family.

\subsection{Bioinformatic analysis}

The presence of the patient's sequence variant in the general population and its frequency was assessed using the following databases:

dbSNP (Single Nucleotide Polymorphisms database) by the National Center for Biotechnology Information (NCBI) (www.ncbi.nlm.nih. gov/projects/SNP/) [12];

Exome Variant Server (NHLBI GO Exome Sequencing Project (ESP), Seattle, WA (http://evs.gs.washington.edu/EVS/);

Exome Aggregation Consortium (ExAC), Cambridge, MA (URL: http://exac.broadinstitute.org), Version 0.3 January 13, 2015.

Prediction analysis of sequence variant effects on the protein structure/function was performed using the following software: Alamut Visual version 2.4 (Alamut Visual version 2.6 (Interactive Biosoftware, Rouen, France, http://www.interactive-biosoftware.com/alamutvisual, including SIFT [sort intolerant from tolerant human protein], Mutation Taster, and Polyphen-2 [Polymorphism Phenotyping v2]).

\subsection{Structural analysis}

The putative structure of the CD25 altered form, the object of this study, has been predicted by Homology Modeling (HM) using SWISSMODEL (http://swissmodel.expasy.org). The p.Cys51Gly IL2R $\alpha$ amino acid sequence was used as target for the HM analysis and the structure of wild type IL2Ra (sushi D1 and D2 domains) as template. The PDB files generated by HM were superimposed with the structure of wild type sushi domains of the human IL2R $\alpha$ structure by PyMOL software (The PyMOL Molecular Graphics System, Version 0.99, DeLano Scientific, LLC, San Carlos, CA).

\section{Results}

\subsection{Lack of surface CD25 protein expression}

The cytofluorimetric analysis of freshly isolated PBMCs from the patient revealed total absence of cell surface $\mathrm{CD} 25$ expression on $\mathrm{CD} 4^{+}$ $\mathrm{T}$ cells (Fig. 1A), while CD25 was detected on $\mathrm{CD} 4^{+} \mathrm{T}$ cells of parents and HC. CD25 surface expression on $\mathrm{CD}^{+}{ }^{+} \mathrm{T}$ cells of the patient remained undetectable upon TCR-mediated activation even in the presence of exogenous IL-2, while CD4 ${ }^{+} \mathrm{T}$ cells of a HC tested in parallel 

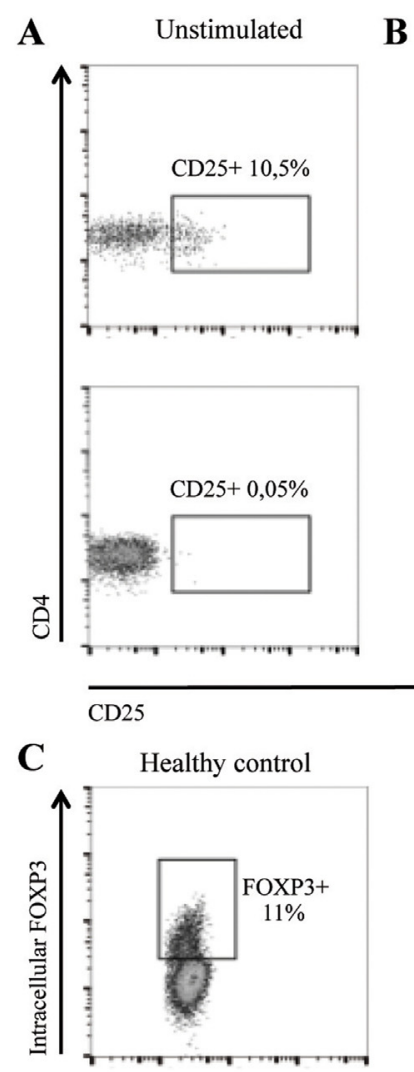

CD4
B Anti-CD3/CD28 mAb
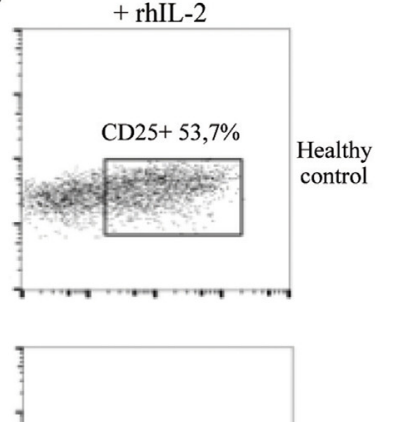

$\begin{array}{ll}\mathrm{CD} 25+0,03 \% & \mathrm{CD} 25\end{array}$

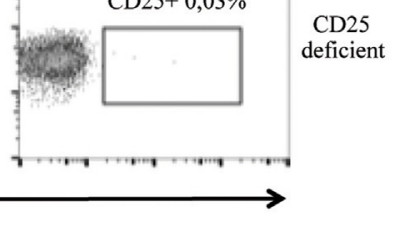

CD25 deficient

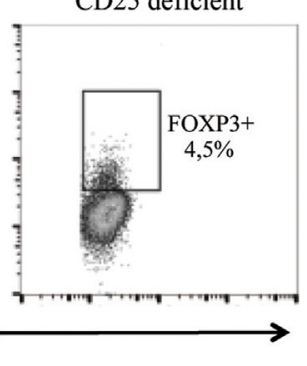

D
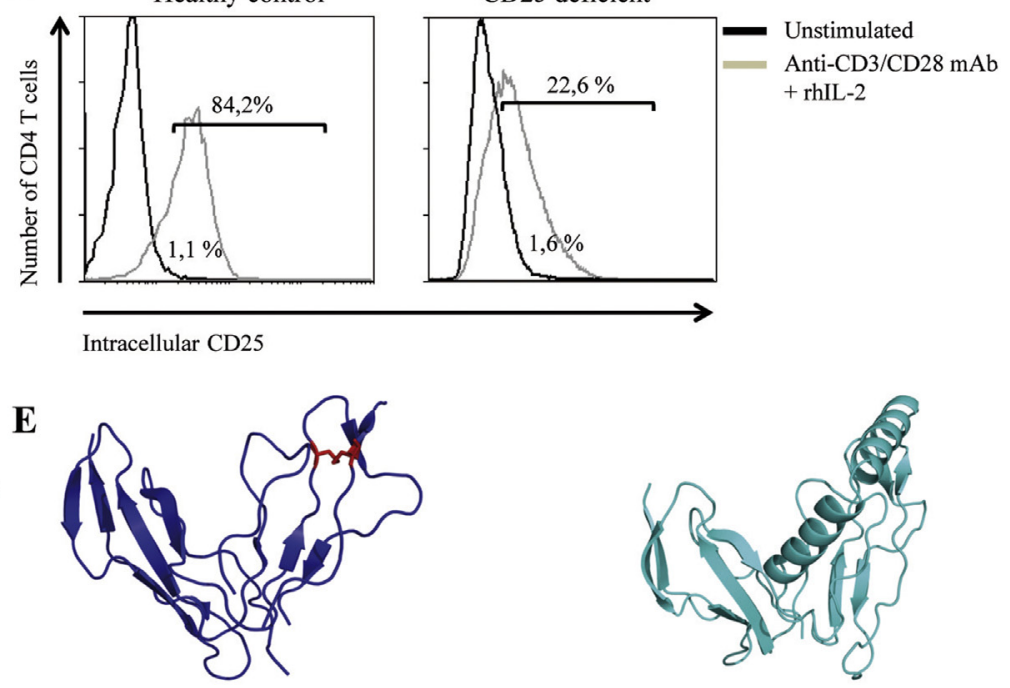

Wild type CD25

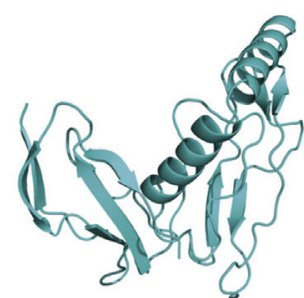

Mutated CD25 (p.Cys51Gly)

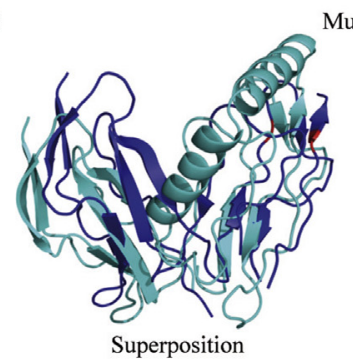

Fig. 1. CD25 expression and protein structure. (A) Surface $\mathrm{CD} 25$ expression on resting $\mathrm{CD}^{+}{ }^{+} \mathrm{T}$ cells in the patient and $\mathrm{HC}$. (B) Surface $\mathrm{CD} 25$ expression on $\mathrm{CD} 4{ }^{+} \mathrm{T}$ cells stimulated with CD3/CD28 T cell expander beads + rhIL2 (100 U/ul) in patient and HC. (C) Intracytoplasmic FOXP3 expression in CD4 ${ }^{+}$T cells in the patient and HC. (D) Intracytoplasmic CD25 expression in $\mathrm{CD}^{+}{ }^{+} \mathrm{T}$ cells stimulated with CD3/CD28 T cell expander beads + rhIL2 (100 U/ml) in patient and HC. Values are expressed as percentage of $\mathrm{CD} 4{ }^{+} \mathrm{T}$ cells. (E) Representative model of altered CD25 putative structure form predicted by Homology Modeling. Wild-type CD25 structure in blue with disulfide bond Cys51-Cys82 in red. Model of p.Cys51Gly CD25 structure in light blue. Superposition of wild-type and model of p.Cys51Gly CD25 structure is shown. (For interpretation of the references to colour in this figure legend, the reader is referred to the web version of this article.)

showed an increase of surface CD25 expression (Fig. 1B). The percentage of FOXP3-expressing CD4 ${ }^{+}$T cells was comparable to HC (Fig. 1C).

Further cytofluorimetric analysis in freshly isolated PBMCs from the patient and HC revealed the presence of intracytoplasmic CD25 expression. In particular $\mathrm{CD} 4^{+} \mathrm{T}$ cells showed a boost in intracytoplasmic $\mathrm{CD} 25$ expression immediately after $\mathrm{T}$ cells activation and in the presence of exogenous IL2, although the patient showed lower levels of CD25 expression compared to HC (Fig. 1D).

\subsection{Novel missense mutation in IL2R $\alpha$ gene}

Molecular analysis of the IL2Ra gene sequence on the patient's gDNA revealed the presence of a previously unreported mutation. The patient resulted homozygous for a c. $151 \mathrm{~T}>\mathrm{G}$ transversion located in exon 2, leading to the substitution of the amino acid Cysteine at codon 51 with a Glycine (p.Cys51Gly) within the Sushi Domain D1 of the protein. The patient's parents proved to be heterozygous for the same mutation. The variation was absent in the 6500 exomes analysed by the Exome Sequencing Project (ESP) and in the 60,700 exomes evaluated by the Exome Aggregation Consortium (ExAC).

The reported crystal structure of the wild-type IL2R $\alpha$ indicates that the cysteine residue at codon 51 is involved in intradomain D1 disulfide-bond formation. Sequence alignment data showed that the residue is highly conserved among species and bioinformatic analysis of the variation indicated that the amino acid change might be deleterious for protein structure.

\subsection{Mutated protein structure instability}

To get better insight into the molecular level of possible effects of the p.Cys51Gly on the protein structure, the putative structure of the altered subunit of CD25 was obtained by HM. In the mutated protein form the disulfide bond Cys51-Cys82, that stabilizes the sushi domain 1, is lost. Four different models of CD25 p.Cys51Gly were obtained by HM analysis. These multiple models computed by HM suggest increased entropy and instability of the protein structure once the disulfide bond is lost (Fig. 1E).

\subsection{Defective proliferative response and cytokine production}

We performed a proliferative assay to evaluate the ability of patient's T cells to respond to polyclonal TCR-mediated stimulation using anti-CD3/CD28 monoclonal antibodies (mAb) and the ability to respond to IL-2 through rhIL-2 stimulation. We also tested the proliferative response to rhIL-15 to asses if the eventual absence to IL-2 response could be partially replaced by the IL- $2 \mathrm{R} \beta$ signalling. The patient's T cells showed a poor response to non-specific TCR activation compared to HC and showed also a mild, dose-dependent proliferation to rhIL-2, although the response was not as vigorous as in HC. Additionally, the patient's T cells response to rhIL-15 was present but poor (Fig. 2A).

Cytokine production showed that patient's cells, when stimulated, were able to produce only IFN- $\gamma(626 \pm 52 \mathrm{pg} / \mathrm{ml})$ compared to not stimulated cells. No significant production of IL-17 or IL-4 was found. 

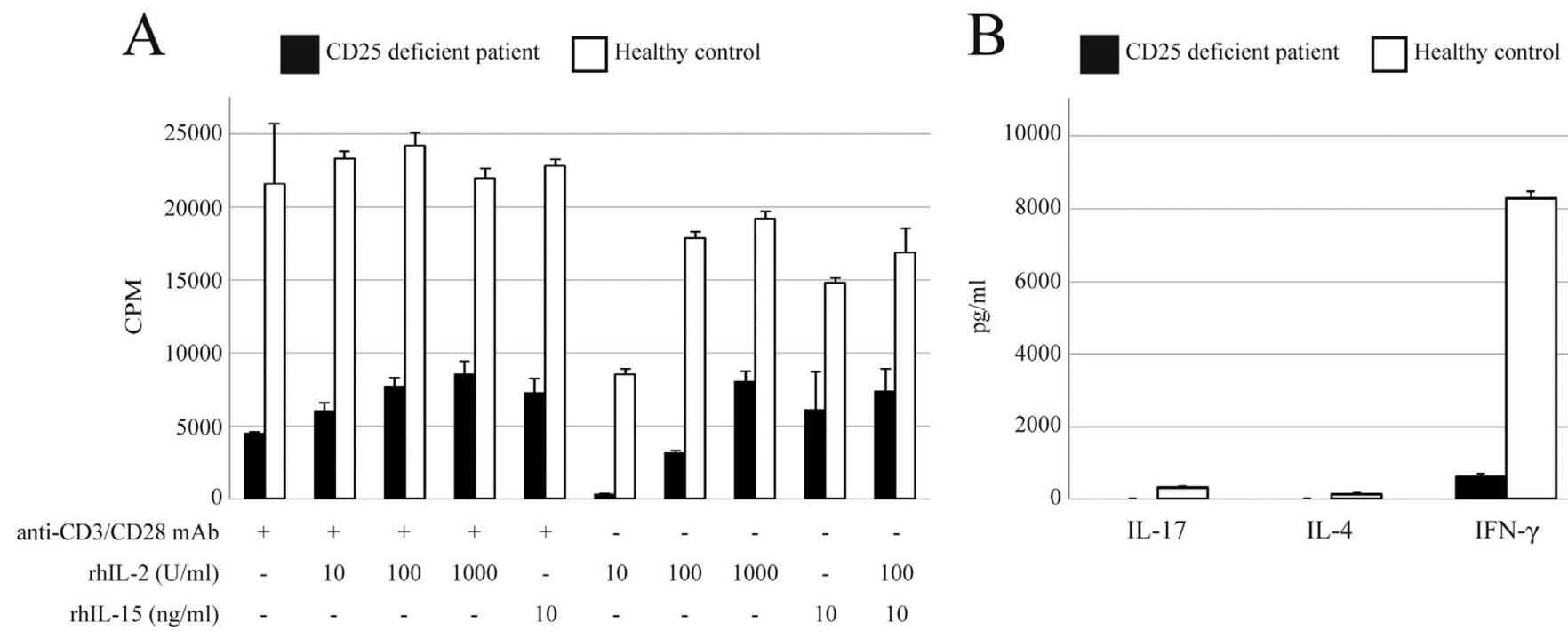

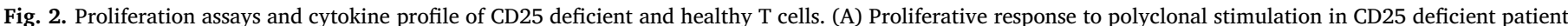

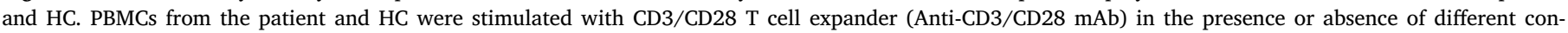

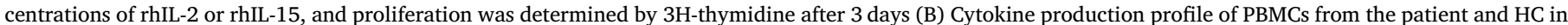
presence of PMA and ionomycin.

Conversely, HC stimulated cells were able to produce all three tested cytokines compared to not stimulated cells and cytokine levels resulted significantly higher than those detected in the patient's cells (IFN- $\gamma$ : $8298 \pm 175 \mathrm{pg} / \mathrm{ml}, \mathrm{IL}-4: 140 \pm 26 \mathrm{pg} / \mathrm{ml}, \mathrm{IL}-17: 315 \pm 33 \mathrm{pg} / \mathrm{ml}$ ) (Fig. 2B).

\section{Discussion}

This paper reports the case of a two-month-old boy presenting with early onset enteropathy and dermatitis resembling IPEX syndrome. Cytofluorimetric analysis revealed the total absence of CD25 cell surface expression. This result quickly addressed the molecular investigation towards the analysis of IL2R $\alpha$ gene sequence, which showed the presence of a novel homozygous missense variation (c.151 T > G; p.Cys51Gly). The sequence variant is predicted to cause the replacement of a Cysteine that is involved in an intra-domain D1 disulfidebond formation. Previous studies of IL2R $\alpha$ crystal structure revealed that the two "sushi-like" domains D1 and D2 are in intimate contact with one another through an extensive inter-domain interface and that intra- and inter-domain disulfide bonds enforce the stability of the structure and the strand exchange [13,14].

To further investigate the effect of IL2R $\alpha$ mutation on protein structure, we analysed the putative configuration of the altered subunit by HM analysis. Four different models were obtained and compared to the wild type protein structure; for all of them the lack of the disulfide bond found in the patient led to a total loss of the correct folding of the protein in sushi 1 domain.

We hypothesise that the disruption of D1 disulfide-bond between Cysteins 51 and 82 due to amino acid change could lead to alteration of the protein structure and to subsequent degradation of the misfolded subunit after its synthesis.

Cells possess a highly active quality control system that recognizes the folding state of proteins, detects terminally misfolded proteins, and specifically eliminates them by proteolysis. The endoplasmic reticulum associated degradation (ERAD) machinery retro-translocate misfolded proteins back to cytosol where the ubiquitin proteasome system (UPS), the major cellular protein elimination machinery in eukaryotic cells, is located [15].

Cytofluorimetric analysis strongly supported our hypothesis revealing the presence of intracytoplasmic CD25 expression in patient's $\mathrm{CD}^{+} \mathrm{T}$ cells, thus confirming that the variation does not abrogate
IL2R $\alpha$ transcription and translation, but shows the absence of cell surface CD25 expression as a result of possible misfolded protein degradation.

The proliferative assay on the patient's PBMCs confirmed an impairment to non-specific TCR activation, as well as to IL2 and IL15 dosedependent proliferation. This is due to the binding of IL2 to IL2 $\beta \gamma$ low affinity complex and of IL15 to the complex IL15R $\alpha$-IL2R $\beta$ that helps to by-pass the lack of IL2. Cytokine studies also confirmed an altered activation profile in the patient compared to HC.

IPEX syndrome, due to FOXP3 mutation, was one of the first diseases of monogenic autoimmunity to be described. Various forms of congenital immunedysregulation, not showing FOXP3 mutations, have been labelled over time and somehow improperly as "IPEX-like" diseases. CD25 deficiency syndrome is a rare autosomal recessive disorder of overwhelming autoimmunity and may be considered the only real phenocopy of IPEX. Prompt treatment with HSCT is the only possible cure of for the disease. Therefore, a timely clinical and molecular diagnosis of CD25 deficiency is mandatory for a successful therapeutic intervention. The patient described in this paper underwent, at three months of age, haploidentical allogeneic HSCT using TCR a/b and CD19 depletion and reduced intensity conditioning (RIC) with treosulfan, fludarabine and antithymocyte globulin (ATG) and anti-CD20 as graftversus-host disease (GvHD) prophylaxis, however no engraftment was attained. A second successful HSCT from matched unrelated donor was performed at seven months of age using a RIC regimen with low-dose busulfan and fludarabine and ATG, metrotexate and ciclosporin as GvHD prophylaxis. The patient showed a reasonable immune reconstitution, a good donor chimerism (95\% donor 5\% recipient) at four months post HSCT, and a complete resolution of the symptoms. He developed a mild skin GvHD which was successfully treated with immune suppression.

This report underlines the importance of cytofluorimetric analysis as a diagnostic tool of CD25 deficiency. As pointed out by our results and in agreement with previous reports in literature, all patients identified to date with $I L 2 R \alpha$ gene mutations have shown total absence or markedly reduced CD25 cell surface expression. As reported by Goudy et al, CD25 protein expression is maintained in the cytoplasm, although at a lower level compared to healthy controls [3]. Immunophenotyping of Tregs can be considered an effective screening tool to discriminate between IPEX and CD25 deficiency syndrome and can neatly address molecular diagnosis. Furthermore, HM is a valuable 
method to predict protein structure in IL2R $\alpha$ mutated subunits and possible effects of gene variants on the protein stability.

In conclusion, a well-defined clinical and immunophenotypic characterization of CD25 deficiency syndrome will allow an early molecular diagnosis and promptly lead to the appropriate treatment of the disease.

Supplementary data to this article can be found online at https:// doi.org/10.1016/j.clim.2019.02.003.

\section{Acknowledgement}

The authors thank the patient and his family for their support and cooperation. We greatly appreciated collaboration with the colleagues of the University-Hospital of Padova, Padova, Italy for proving us patient's clinical information and follow-up.

\section{Funding}

University of Florence, internal funding (ex-60\% ateneo).

\section{Conflict of interest}

The authors declare no conflict of interest.

\section{References}

[1] N. Sharfe, H.K. Dadi, M. Shahar, C.M. Roifman, Human immune disorder arising from mutation of the alpha chain of the interleukin-2 receptor, Proc. Natl. Acad. Sci. U. S. A. 94 (1997) 3168-3171, https://doi.org/10.1073/pnas.94.7.3168.

[2] A.A. Caudy, S.T. Reddy, T. Chatila, J.P. Atkinson, J.W. Verbsky, CD25 deficiency causes an immune dysregulation, polyendocrinopathy, enteropathy, X-linked-like syndrome, and defective IL-10 expression from CD4 lymphocytes, J. Allergy Clin. Immunol. 119 (2007) 482-487, https://doi.org/10.1016/j.jaci.2006.10.007.

[3] K. Goudy, D. Aydin, F. Barzaghi, E. Gambineri, M. Vignoli, S.C. Mannurita, C. Doglioni, M. Ponzoni, M.P. Cicalese, A. Assanelli, A. Tommasini, I. Brigida, R.M. Dellepiane, S. Martino, S. Olek, A. Aiuti, F. Ciceri, M.G. Roncarolo, R. Bacchetta, Human IL2RA null mutation mediates immunodeficiency with lymphoproliferation and autoimmunity, Clin. Immunol. 146 (2013) 248-261, https:// doi.org/10.1016/j.clim.2013.01.004.
[4] L. Bezrodnik, M.S. Caldirola, A.G. Seminario, I. Moreira, M.I. Gaillard, Follicular bronchiolitis as phenotype associated with CD25 deficiency, Clin. Exp. Immunol. 175 (2014) 227-234, https://doi.org/10.1111/cei.12214.

[5] T.L. Roth, C. Puig-Saus, R. Yu, E. Shifrut, J. Carnevale, P.J. Li, J. Hiatt, J. Saco, P. Krystofinski, H. Li, V. Tobin, D.N. Nguyen, M.R. Lee, A.L. Putnam, A.L. Ferris, J.W. Chen, J.N. Schickel, L. Pellerin, D. Carmody, G. Alkorta-Aranburu, D. Del Gaudio, H. Matsumoto, M. Morell, Y. Mao, M. Cho, R.M. Quadros, C.B. Gurumurthy, B. Smith, M. Haugwitz, S.H. Hughes, J.S. Weissman, K. Schumann, J.H. Esensten, A.P. May, A. Ashworth, G.M. Kupfer, S.A.W. Greeley, R. Bacchetta, E. Meffre, M.G. Roncarolo, N. Romberg, K.C. Herold, A. Ribas, M.D. Leonetti, A. Marson, Reprogramming human T cell function and specificity with non-viral genome targeting, Nature 559 (2018) 405-409, https://doi.org/10. 1038/s41586-018-0326-5.

[6] S.A.A. Nashat Al Sukaitia*, Aisha Al Sinanib, Suad Al Ismailyc, Samiuddin Shaikhc, Pulmonary hemorrhage in a case of CD25 deficiency, LymphoSign J. 1 (2014) 39-43.

[7] X. Wang, M. Rickert, K.C. Garcia, Structure of the quaternary complex of interleukin-2 with its $\alpha, \beta$ and $\gamma$ creceptors, Science (80-.). 310 (2005) 1159-1163, https://doi.org/10.1126/science.1117893.

[8] S. Sakaguchi, Naturally arising CD4 + regulatory t cells for immunologic self-tolerance and negative control of immune responses, Annu. Rev. Immunol. 22 (2004) 531-562, https://doi.org/10.1146/annurev.immunol.21.120601.141122.

[9] W.E. O'Gorman, H. Dooms, S.H. Thorne, W.F. Kuswanto, E.F. Simonds, P.O. Krutzik, G.P. Nolan, A.K. Abbas, The initial phase of an immune response functions to activate regulatory T cells, J. Immunol. 183 (2009) 332-339, https://doi.org/10. 4049/jimmunol.0900691.

[10] E. Zorn, E.A. Nelson, M. Mohseni, F. Porcheray, H. Kim, D. Litsa, R. Bellucci, E. Raderschall, C. Canning, R.J. Soiffer, D.A. Frank, J. Ritz, IL-2 regulates FOXP3 expression in human CD4 + CD25 + regulatory T cells through a STAT-dependent mechanism and induces the expansion of these cells in vivo, Blood 108 (2006) 1571-1579, https://doi.org/10.1182/blood-2006-02-004747.

[11] N. Capitani, A. Amedei, S.R. Paccani, A. Matucci, A. Vultaggio, G. Del Prete, C.T. Baldari, M.M. D'Elios, Impaired TH2 response in patients with Vav1-deficient common variable immunodeficiency with T-cell defects, J. Allergy Clin. Immunol 126 (2010) 671-675, https://doi.org/10.1016/j.jaci.2010.05.033.

[12] K.S.S.T. Sherry, M.H. Ward, M. Kholodov, J. Baker, L. Phan, E.M. Smigielski, dbSNP: the NCBI database of genetic variation, Nucleic Acids Res. 29 (2001) 308-311, https://doi.org/10.1093/nar/29.1.308.

[13] M. Rickert, X. Wang, M.J. Boulanger, N. Goriatcheva, K.C. Garcia, The Structure of Interleukin-2 Complexed with its Alpha Rece, Vol. 2057 (2005), pp. 1477-1481.

[14] D.J. Stauber, E.W. Debler, P.A. Horton, K.A. Smith, I.A. Wilson, Crystal structure of the IL-2 signaling complex: paradigm for a heterotrimeric cytokine receptor, Proc. Natl. Acad. Sci. 103 (2006) 2788-2793, https://doi.org/10.1073/pnas. 0511161103.

[15] M.M. Hiller, A. Finger, M. Schweiger, D.H. Wolf, ER degradation of a misfolded luminal protein by the cytosolic ubiquitin-proteasome pathway, Science (80-.). 273 ${ }^{1}$ (1996) 1725-8. doi:https://doi.org/10.1126/science.273.5282.1725. 